\title{
Association of Fusobacterium nucleatum in the Progression of Colorectal Cancer
}

\section{Asma Rafique, Young-Sang Koh*}

Department of Microbiology and Immunology, College of Medicine, and Jeju Research Center for Natural Medicine, Jeju National University, Jeju 63243, Republic of Korea

Corresponding

Young-Sang Koh, Professor

Department of Microbiology and Immunology, College of Medicine, Jeju National University, 102 Jejudaehakno, Jeju 63243, Republic of Korea

Phone : +82-64-754-3851

Fax : +82-64-702-2687

E-mail : yskoh7@jejunu.ac.kr

Received : April 30, 2021

Revised : June 10, 2021

Accepted : June 11, 2021

No potential conflict of interest relevant to this article was reported.

Copyright (C) 2021 Journal of Bacteriology and Virology

(C) This is an Open Access article distributed under the terms of the Creative Commons Attribution Non-Commercial

License

(http://creativecommons.org/

license/by-nc/3.0/).
Colorectal cancer (CRC) is recognized as the third most common malignancy worldwide. Previous studies indicated several genetic and environmental factors that potentiate CRC. However, the role of microbiota in the etiology of CRC is becoming a major concern nowadays. Fusobacterium nucleatum (F. nucleatum), an anaerobic bacterium, mostly resides in the oral cavity and is a causative agent of various oral inflammatory diseases. It is evident from the recent studies that $F$. nucleatum is present in abundance in the CRC tissues as compared to the adjacent normal tissues. F. nucleatum has certain virulence factors like FadA and Fap2 which help in its adhesion to the mucosal cells and promotes carcinogenesis. Besides that, these virulence factors induce cytokine production and recruitment of inflammatory cells, hence it creates an environment favorable for neoplastic growth. Furthermore, $F$. nucleatum can suppress the host immune system by interfering with normal functions of macrophages, dendritic cells, $T$ cells, tumor-associated neutrophils and natural killer cells. It was also revealed that CRC patients with high numbers of $F$. nucleatum showed drug resistance and cancer recurrence. From the previous investigations, it was concluded that $F$. nucleatum has a diagnostic as well as prognostic value for CRC patients. In the present study, we have outlined the current data on the detection methods, pathogenic mechanisms, immunosuppression effects and clinical management of $F$. nucleatum-associated CRC.

Key Words: Colorectal cancer, Fusobacterium nucleatum, Virulence factors, Carcinogenesis, Immunosuppression

\section{INTRODUCTION}

Colorectal cancer (CRC) is the most prevalent malignancy of the digestive tract, which is currently viewed as a serious health issue in the world (1). Overall, CRC is considered as the third most common cancer in males while in females it is ranked as the second most commonly occurring neoplasm (2). CRC is a multifactorial complex disease, which is influenced by a number of risk factors like the family history of CRC, diet, lifestyle and obesity, while inflammation is recognized as a crucial risk factor (3).

Human gut microbiota is comprised of approximately $10^{14}$ microorganisms which play a significant role in the maintenance of human health. These microbes perform a number of functions to regulate intestinal homeostasis, including host metabolism, mucosal barrier integrity and immunomodulation $(4,5)$. Several studies have confirmed that dysbiosis of microbiota is involved in the pathogenesis 
of various diseases specifically inflammatory bowel disease (IBD), neuropsychiatric disorders, cardiometabolic diseases and cancer (6).

Recent data showed a close relationship between particular bacterial species (Bacteroides fragilis, Escherichia coli, Streptococcus gallolyticus, Prevotella intermedia, Streptococcus bovis, Fusobacterium nucleatum and Enterococcus faecalis) and CRC (7-14). Furthermore, it is evident from the previous report that the number of Fusobacterium nucleatum ( $F$. nucleatum) is much higher in CRC patients as compared to healthy individuals (15). F. nucleatum promotes CRC through multiple mechanisms: including E-cadherin/ $\beta$-catenin signaling (16), TLR4/MyD88 signaling (17), autophagy activation (18) and by suppressing host immunity (19) etc. (see the later section), while well-known anaerobic bacteria enterotoxigenic Bacteroides fragilis (ETBF) secretes Bacteroides fragilis-derived toxin (BFT) which can cause inflammation through Th17 cells $(20,21)$. Furthermore, BFT is involved in the upregulation of spermine oxidase (SPO) and generation of reactive oxygen species (ROS), resulting in DNA damage and promotion of intestinal tumorigenesis (22).

F. nucleatum is a gram-negative anaerobic bacterium that mostly presents in the oral cavity and intestine. Inside the oral cavity $F$. nucleatum can cause oral inflammation and is responsible for certain diseases like periodontitis and gingivitis (23-25), while it is also associated with brain abscesses, pancreatic cancer, liver abscesses and premature births (26-29). F. nucleatum is the most prevalent bacterial species found in CRC tissues and its relevancy in CRC has been supported by a number of pre-clinical and clinical studies $(9,30-32)$. Previous studies have also confirmed that $F$. nucleatum associates with some anaerobic bacteria including Streptococcus, Leptotrichia and Campylobacter spp. which synergistically promotes the occurrence of $\operatorname{CRC}(33,34)$. Metagenomic analysis using whole-genome sequencing (30), transcriptome sequencing (35) and bacterial $16 \mathrm{~S}$ ribosomal RNA (rRNA) sequencing (36) have shown enrichment of $F$. nucleatum in CRC relative to adjacent normal tissues. In comparison to Bacteroidetes and Firmicutes, the levels of $F$. nucleatum shown to be high in CRC tissues $(30,31)$. Additionally, several studies have documented that the number of $F$. nucleatum increases in the tumor and fecal samples of CRC patients as compared to healthy cohorts $(16,32,35,37-40)$. It was indicated that the presence of $F$. nucleatum in CRC is associated with the poor prognosis of disease and also promotes chemoresistance $(18,41)$. Actually, a high burden of $F$. nucleatum triggers a number of molecular events that are associated with CRC carcinogenesis, specifically microsatellite instability (MSI), CpG island methylator phenotype (CIMP) and tumorigenic mutations in TP53, BRAF, CHD7 and $C H D 8$ genes $(41,42)$. The notion that $F$. nucleatum has a role in CRC tumorigenesis was first proved in $A p C^{\text {min/ } /}$ mice (19), which was later supported by the findings that this bacterium promotes CRC by stimulating $\beta$-catenin pathway through FadA adhesin (43). Previous studies revealed that in comparison to $F$. nucleatum-negative cases, $F$. nucleatum-positive cases are associated with more advanced stages of CRC, proximal tumor location, MSI-high, CIMP-high and lower density of $\mathrm{CD}^{+} \mathrm{T}$ cells $(9,39,44)$. Metagenomics analysis indicate that $F$. nucleatum is associated with human CRC, but whether this is an indirect or causal link remains unclear (45). F. nucleatum was previously regarded as a passenger bacterium in human intestinal tract $(45,46)$. Recently, it has been considered to be a potential initiator of CRC susceptibility $(38,42)$. It was further observed that $F$. nucleatum promotes colorectal tumorigenesis in $A p C^{\mathrm{min} / t}$ mice (19). Together, these studies shed light on the fact that $F$. nucleatum plays an important role in the initiation, promotion and progression of tumor cell growth in CRC, supporting that $F$. nucleatum is the causal factor of CRC instead of a consequence.

Aiming to support the future research on $F$. nucleatum and to unveil new diagnostic and therapeutic possibilities for CRC, the present study will focus on the following topics in detail: methods used for the detection and quantification of $F$. nucleatum in CRC; pathogenesis of $F$. nucleatum in CRC; $F$. nucleatum promotes epigenetic changes in CRC; $F$. nucleatum suppresses host immunity in CRC; role of $F$. nucleatum in drug resistance; screening and prevention strategies of $F$. nucleatum-associated CRC; and clinical management of $F$. nucleatum in CRC patients.

\section{DETECTION METHODS OF F. NUCLEATUM IN CRC}

Previous studies have used various techniques for detecting $F$. nucleatum in CRC like such as quantitative real-time 
polymerase chain reaction ( $\mathrm{PPCR}$ ), fluorescent quantitative polymerase chain reaction (FQ-PCR), droplet digital polymerase chain reaction (ddPCR), enzyme-linked immunosorbent assay (ELISA) and fluorescence in situ hybridization (FISH) (47). In FQ-PCR method, Taqman (flurorescent) probe specific for target gene is used while in conventional qPCR, SYBR Green dye is used (48). Also, researchers detected $F$. nucleatum in different samples based on whole genome sequencing, CRC frozen tissues, feces, and formalin-fixed paraffin-embedded (FFPE) CRC tissues (47).

A study conducted on Chinese CRC patients used FQ-PCR and FISH methods to detect $F$. nucleatum, which revealed that 88 out of $101(87.1 \%)$ cases had increased levels of $F$. nucleatum in frozen and FFPE tissues respectively (37). Two American studies measured $F$. nucleatum abundance in FFPE tissue by qPCR, in which 76/598 (13\%) CRC patients exhibited positive response (39). Similarly, one Japanese research enrolled 511 CRC patients and detected $F$. nucleatum in $44(8.6 \%)$ individuals from CRC FFPE tissues by the aid of GPCR (44). In addition to that, another Japanese study used qPCR to estimate the concentration of $F$. nucleatum from the DNA of patient's CRC tissues, detected its presence in 111 out of $149(74 \%)$ cases (42). One more Japanese research reported regarding $F$. nucleatum enrichment in CRC FFPE tissues of $286 / 511$ (56\%) patients by qPCR (16). Similar results were obtained in stool samples of Japanese CRC patients by ddPCR method, where 85/158 (54\%) individuals had high levels of $F$. nucleatum (49). Also, researchers determined $F$. nucleatum by qPCR in FFPE tissues obtained from CRC patients and stated that it is abundant in colon cancers as compared to rectal cancers (50). Thus, the concentration of $F$. nucleatum steadily decreases from cecum to rectum.

In comparison to $\mathrm{APCR}$, ddPCR and FQ-PCR are regarded as more efficient and convenient methods of detecting low bacterial concentrations $(51,52)$. Scientists extracted DNA from frozen CRC and normal tissues and analyzed through whole-genome sequencing (30). It was revealed that CRC tissues were enriched with $F$. nucleatum as compared to normal tissues (30). Similar results were obtained in another study based on transcriptome sequencing, where $F$. nucleatum were present in abundance in CRC tissues as compared to adjacent normal tissues (35).

\section{POTENTIAL ROLE OF F. NUCLEATUM IN CRC}

The underlying pathogenic mechanisms of $F$. nucleatum in CRC have been studied by various researchers (Fig. 1) (53, 54). It has been demonstrated that three virulence factors of $F$. nucleatum play a critical role in the pathogenesis as well as metastasis of CRC. These factors are: fusobacterium adhesin A (FadA), lipopolysaccharides (LPS) and fusobacterium autotransporter protein 2 (Fap2), all of which are present on the outside of $F$. nucleatum (55-57). It is well known that the invasion of specific bacterium into the epithelial cells is the prerequisite step of $F$. nucleatum-positive CRC (58). FadA is a highly conserved virulence factor for $F$. nucleatum, that facilitates its attachment to both cancerous and non-cancerous cells (43). Interestingly, there are two forms of FadA : one is known as pre-FadA which anchors to the membrane and consists of 129 amino acids, while the other form is mature FadA which consists of 111 amino acids $(59,60)$. Additionally, when these two forms are mixed together then active FadA is formed, which has a potential role in the attachment and invasion into host epithelial cells (43). FadA binds to E-cadherin which is present in normal as well as CRC cells (16). Actually, E-cadherin has tumor suppressive properties, but FadA binding inhibits this function and stimulates the proliferation of CRC cells (61). E-cadherin is a glycoprotein, composed of an extracellular, transmembrane and a cytoplasmic domain. Cytoplasmic domain further binds to various cytosolic components, particularly $\beta$-catenin. FadA binds to E-cadherin, resulting in the phosphorylation and internalization of E-cadherin, that leads to its degradation. Loss of E-cadherin is not only associated with the reduction of cell-cell adhesive properties but also increases the cytoplasmic pool of $\beta$-catenin. Thus, $\beta$-catenin migrates into the nucleus and results in the activation of $\beta$-catenin-regulated transcription (CRT). This CRT promotes the genetic expressions of various genes such as oncogenes (c-myc, Ccnd1), wnt genes (wnt7a, wnt7b, wnt9a), genes encoding transcription factors (LEF-1, NF-kB, TCF1, TCF3, TCF4) and inflammatory cytokines (IL-6, IL-8, IL-18) (16). CRC tissues highly express D-galactose- $\beta-(1-3)-N$-acetyl-D-galactosamine (Gal-GalNac) polysaccharide to which another virulence factor Fap2 binds, that leads to enrichment of $F$. nucleatum in CRC tissues (62). Another research 


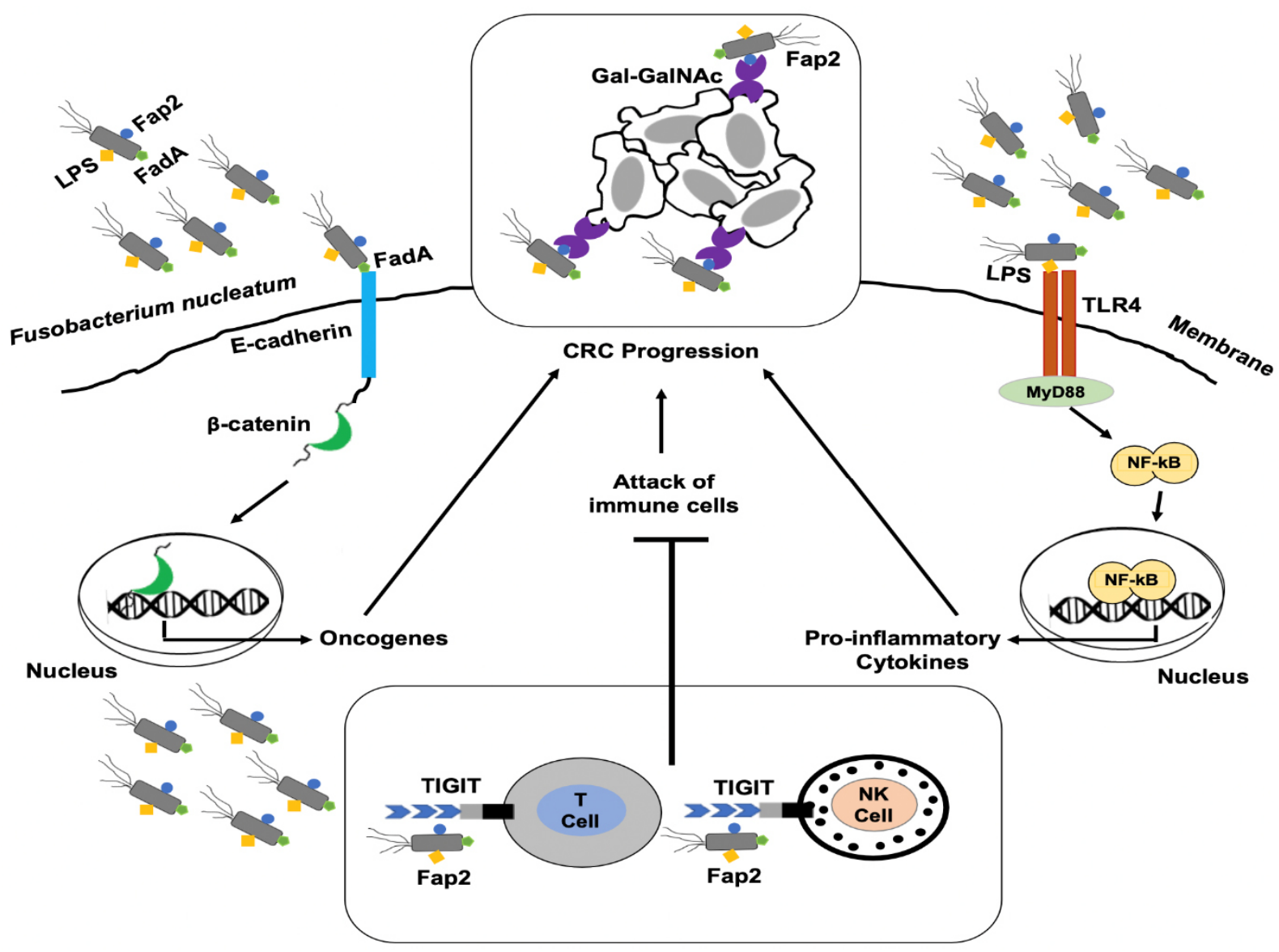

Fig. 1. Pathogenic mechanisms of $F$. nucleatum in CRC. F. nucleatum invades into the epithelial cells using virulence factors such as FadA, Fap2 and LPS. FadA attaches to the E-cadherin and activates the $\beta$-catenin signaling pathway, which promotes the expression of oncogenes. F. nucleatum recruits itself to the tumor sites by binding of Fap2 to Gal-GalNAc present on tumor cells. Additionally, Fap2 binds to the TIGIT receptor present on T and NK cells, that leads to inhibit their antitumor activity. Binding of LPS to TLR4 mediates TLR4/MyD88 signaling which leads to the translocation of NF-kB into the nucleus and results in the production of proinflammatory cytokines. Hence, all of these mechanisms potentiate the development and progression of CRC. CRC, colorectal cancer; FadA, fusobacterium adhesin A; Fap2, fusobacterium autotranporter protein 2; Gal-GalNAC, D-galactose- $\beta$-(1-3)-N-acetyl-D-galactosamine; LPS, lipopolysaccharides; MyD88, myeloid differentiation primary response 88; NF-kB, nuclear factor-kB; NK, natural killer; TIGIT, T cell immunoreceptor with Ig and tyrosine-based inhibitory motif domains; TLR4, toll-like receptor 4.

indicated that Fap2 is involved in the binding of $F$. nucleatum to T cell immunoreceptor with $\mathrm{Ig}$ and tyrosine-based inhibitory motif domains (TIGIT). Actually, TIGIT is an immune checkpoint receptor, which is present in NK and T cells. TIGIT has the potency to bind to multiple ligands (CD155 and CD112) present on antigen presenting and tumor cells as well as Fap2 (63). So, Fap2 binding can cause the phosphorylation of TIGIT receptor and inhibits the PI3k, MAPK and NF-KB signaling pathways (63). The inhibition of these pathways strongly reduces the cytotoxic activity associated with NK and $T$ cells, and promotes tumor progression (64). Moreover, another study has manifested that $F$. nucleatum activates the TLR4/MyD88 signaling pathway by LPS, which results in the activation of NF-kB. NF-kB induces the expression of various proinflammatory genes that result in the production of inflammatory cytokines such as IL-1, IL-2, IL-6, IL-8 and IL-12. These cytokines create an inflammatory environment that promotes the progression of CRC (17). In summary, such studies based on F. nucleatum virulence factors provide new vision for the better understanding of $F$. nucleatum-associated CRC. 


\section{F. NUCLEATUM PROMOTES EPIGENETIC CHANGES IN CRC}

It has been reported that $F$. nucleatum takes part in the epigenetic modifications of CRC. Previous findings have shown that $F$. nucleatum in CRC is associated with certain molecular features like high microsatellite index (MSI), CpG island methylator phenotype (CIMP) status, hypermethylation in the promoter region of mismatch repair protein (MLH1) and enhanced expression of miRNA-21 (42). Moreover, all these phenotypic changes mostly occur in the ascending colon of the gastrointestinal tract, which is also the major site for the residence of $F$. nucleatum $(65,66)$. These studies suggest that there is a close association between $F$. nucleatum and CRC microenvironment.

In case of CIMP, there is a hypermethylation of various $\mathrm{CpG}$ islands present in the promoter regions of several genes. Such increased methylation of the $\mathrm{CpG}$ island will lead to inactivation of tumor suppressor genes and promotion of chronic inflammation (67). MSI accounts for about $15-20 \%$ cases of CRC and is attributed to mutations in the DNA mismatch repair genes (42). F. nucleatum is responsible for increased production of reactive oxygen species and inflammatory cytokines, both of which contribute to the epigenetic silencing of the MLH1 and lead to MSI tumors (44).

miRNAs are recognized as small non-coding RNAs that function in RNA silencing as well as in the regulation of post-transcriptional gene expression. Also, miRNAs are now considered as diagnostic and therapeutic biomarker for a number of diseases, especially cancer (68). Previous data have proved that $F$. nucleatum might take part in increasing the levels of miRNA-21 through epigenetic regulation (32). Furthermore, miRNA-21 can increase the levels of IL-10 and prostaglandin E2 (PGE2), which suppress antitumor immunity mediated by T cells in the tumor microenvironment $(69,70)$. Therefore, high levels of miRNA-21 are usually associated with poor clinical outcomes (71). Further studies are required to clarify the underlying mechanisms responsible for $F$. nucleatum mediated epigenetic changes in the development of CRC.

\section{F. NUCLEATUM AND HOST IMMUNITY}

Several studies have proved that $F$. nucleatum assists CRC progression by regulating the tumor immune microenvironment $(19,72)$. Increased infiltration of $C D 11 b^{+}$myeloid cells was observed in $A p c^{\min / t}$ mice that fed with $F$. nucleatum. Tumor-associated macrophages (TAMs), M2-like TAMS, dendritic cells, regulatory T cell (Treg) and T helper cell 17 (Th17) also increased in this mice model. Myeloid-derived suppressor cells (MDSC) subsets include monocytic and granulocytic cells which can suppress $\mathrm{CD}^{+} \mathrm{T}$ cells and activate NF-kB driven pro-inlammatory response. These results suggested that there is a link between $F$. nucleatum abundance and immunity in CRC microenvironment (19). F. nucleatum suppresses the host immune system through the following mechanisms:

\section{TAMs}

Many researchers have confirmed that the infiltration of TAMs increased in F. nucleatum-associated CRC (73). Moreover, $F$. nucleatum has a role in M2 polarization and promotes the progression of CRC through TLR4/STAT3 signaling pathway (74). F. nucleatum significantly produce butyric acid as the end product which can cause apoptosis of various immunoregulatory cells such as macrophages and lymphocytes (75). F. nucleatum is able to invade and survive in macrophages and creates the toxic microenvironment by promoting the expression of host indoleamine 2,3-dioxygenase (IDO). Additionally, this IDO disrupts the function of $\mathrm{T}$ lymphocytes and allows $F$. nucleatum-infected macrophages to escape from the attack of cytotoxic T lymphocytes (76).

\section{T cells}

The virulence factors of $F$. nucleatum have been known for their role in the inhibition of T cell activity $(64,77)$. It was 
observed that lower number of $\mathrm{CD}^{+}$and $\mathrm{CD}^{+} \mathrm{T}$ cells are present in $F$. nucleatum enriched $\mathrm{CRC}$ tissues (78). F. nucleatum is responsible for this reduction in T cell number by inhibiting proliferation while promoting apoptosis of T cells (79-81). For instance, F. nucleatum can do so through its inhibitory proteins that induce G1 phase arrest of the cell cycle (80). F. nucleatum promotes $C R C$ by the release of short peptides along with short-chain fatty acids which subsequently cause the recruitment of various cells, particularly MDSCs. Then these MDSCs suppresses the function of $C D 4^{+} \mathrm{T}$ helper cell and hence, further contributes in CRC tumorigenesis (82). Moreover, F. nucleatum virulence factors such as Fap2 and RadD can cause the death of human lymphocytes (77). In CRC, Fap2 can also inhibit the activity of T cells by directly binding to TIGIT, an immunoreceptor present on some T cells (64).

\section{Other immune cells}

All of the NK cells express TIGIT receptor which $F$. nucleatum binds to through Fap2. As a result of this binding, the killing function of NK cells is inhibited and promotes tumor progression (83). One of the studies has demonstrated that tumors from $F$. nucleatum-fed mice are rich in $\mathrm{CD}_{103^{+}}$dendritic cells as compared to the mice fed on a normal diet (19). Additionally, these dendritic cells can enhance the infiltration of $\mathrm{Foxp}^{+}{ }^{+}$regulatory $T$ cells which promote tumor growth by inhibiting the function of cytotoxic T cells (84). The infiltration of tumor associated neutrophils is more in $F$. nucleatum-fed mice in comparison to the control group (19). Recently it was indicated that the tumor associated neutrophils regulate antitumor immunity and have a role in the progression of CRC (72). These studies proposed that $F$. nucleatum is responsible for immune suppression and is associated with a poor prognosis of the disease (85).

\section{ROLE OF F. NUCLEATUM IN DRUG RESISTANCE}

Previous data shed light on the fact that $F$. nucleatum potentiates the development of tumor microenvironment. Drug resistance is a common problem which occurs during the CRC treatment, including either chemotherapy or targeted therapy $(86,87)$. Studies have shown that tumor microenvironment has an impact on drug resistance. Especially in case of CRC, a huge diversity of gut microbiota also has the potential to effect this tumor microenvironment (88). In this manner, F. nucleatum might play a role in promoting drug resistance by regulating tumor microenvironment of CRC. F. nucleatum potentiates drug resistance by the following mechanisms: first, it can mediate autophagy through TLR4 receptors. TLR4/MyD88 signaling pathway is activated by $F$. nucleatum, resulting in the inhibition of miRNA-18a and miRNA-4802. It was observed that miRNA-18a and miRNA-4802 negatively regulate specific autophagy related genes encoding such as Unc-51 like autophagy activating kinase (ULK1) and autophagy-related protein 7 (ATG7), respectively. Hence, the inhibition of these miRNAs by $F$. nucleatum promotes the induction of autophagy related proteins. Thus, increase in autophagy activity can inhibit the chemotherapy-induced apoptosis of CRC cells, that leads to chemoresistance (18). Second, $F$. nucleatum can enhance the chemoresistance of antiangiogenic agents such as bevacizumab. In CRC, the prominent role of F. nucleatum is to secrete proinflammatory cytokines (IL-6 and IL-8) that subsequently contribute in the pro-angiogenesis pathways (89). Third, F. nucleatum promotes the infiltration of myeloid derived suppressor cells, which are also known to contribute in chemoresistance $(90,91)$. F. nucleatum-mediated drug resistance can be reduced by the use of antimicrobial therapy.

\section{SCREENING AND PREVENTION STRATEGIES OF F. NUCLEATUM-ASSOCIATED CRC}

Identifying colorectal adenomas or early adenocarcinomas by screening can effectively reduce CRC mortality $(92,93)$. F. nucleatum can be used as a tool for diagnosis of CRC or for risk stratification in CRC screening (94). For screening of 
F. nucleatum-associated CRC, it is not feasible to obtain tissue samples while the more convenient way is to draw samples from serum and feces. It was mentioned in several studies that $F$. nucleatum infection leads to elevated levels of certain antibodies particularly anti- $F$. nucleatum (Fn)-lgA in the serum of CRC patients $(95,96)$. The combination of detection methods for anti-Fn-IgA with carcinoembryonic antigen and carbohydrate antigen 19-9 enhances the sensitivity to diagnose CRC in its early stages. These findings suggest that the marked increase in serum anti-Fn-IgA antibodies, act as potential marker for early detection of CRC cases (96). Moreover, another recent research used ELISA to measure the levels of antibodies against $F$. nucleatum in the serum samples of CRC patients and healthy individuals. It was observed that high levels of antibodies were present in $F$. nucleatum-positive CRC cases (95). Another non-invasive screening method includes fecal immunochemical test (FIT), but is not recommended for the detection of advanced adenomas due to less sensitivity (97). Later on it was observed that GPCR increase the sensitivity of FIT to 38.6 and $92.3 \%$ for advanced adenomas and CRC, respectively (40). One of the recent research has used qPCR to quantify $F$. nucleatum in stool samples, which manifested that levels of $F$. nucleatum in stool samples were significantly higher in the CRC group as compared with control and polyp group (98). Another study used ddPCR assay for detecting $F$. nucleatum in stool samples from Japanese population. It was concluded from results that in comparison to control group, F. nucleatum levels were significantly higher in the non-advanced adenoma group, advanced adenoma group and CRC group (49). Moreover, a loop-mediated isothermal amplification method has 10-fold more sensitivity as compared to qPCR which basically detect FadA virulence factor (99). So, in the future $F$. nucleatum can be used as a diagnostic marker for the early detection of CRC.

Numerous studies have been conducted to find the impact of diet on the prevalence of CRC (100, 101). It has been proved that diet high in grains, fruits and vegetables decrease the risk of CRC while a western-style diet rich in meat and fats increase its chances (102-104). High-fat-diet along with oncogene activation dampened paneth-cell mediated immunity and thus shift bacterial communities in such a way that promotes intestinal cancer (105). Although, the underlying reason for the contribution of diet in CRC prevalence is not clear, it has believed that gut flora might play an intermediate part in this association. Diet alters the human gut microbiome and long-term dietary patterns allow only specific microbes to retain in the gut while the others may become extinct $(106,107)$.

Previous studies focused on the reduction of $F$. nucleatum in CRC through diet modification (108-110). Diets rich in whole grains and dietary fiber are associated with a lower risk for $F$. nucleatum-positive colorectal cancer but not $F$. nucleatum-negative cancer, supporting a potential role for intestinal microbiota in mediating the association between diet and colorectal neoplasms (110). Diet may influence CRC risk by modulating F.nucleatum abundance as a dietary intervention study noted a marked increase in stool F.nucleatum levels after individuals were switched from a low-fiber to high-fat diet (109). Certain proinflammatory diets elevate the levels of IL-6, TNF receptor superfamily 1B (TNFRSF1B) and creatine protein (CRP), all of which contribute to systemic inflammation (101). Empirical Dietary Inflammatory Pattern (EDIP) score has been mostly used to determine the inflammatory effects of various diets. EDIP score has been assessed from eighteen foods that are known to elevate circulating levels of IL-6, TNFRSF1B and CRP (108). A previous study indicated that elevated EDIP scores are linked with high risk of $F$. nucleatum-associated CRC (100).

\section{CLINICAL MANAGEMENT OF F. NUCLEATUM-ASSOCIATED CRC}

F. nucleatum invades into the epithelial cells and release various cytokines which contribute to the development of tumor microenvironment and promote CRC progression $(17,111-113)$. As inflammation is one the causes of CRC occurrence, so anti-inflammatory agents have been considered to be good candidates for the prevention and treatment of CRC (114). Prostaglandin-Endoperoxide Synthase 2 (PTGS2) is an enzyme which is sufficiently expressed in several types of cancers, including CRC (115). Non-steroidal antiinflammatory drugs (NSAIDs) have been mostly prescribed to inhibit the activity of PTGS2 (116). Moreover, it was demonstrated that $F$. nucleatum enhances the production of this enzyme and promotes inflammatory cascade (111). Hence, it is possible to prevent the occurrence of CRC by the use of NSAIDs. In addition, 
sulindac, a NSAID, has been reported for the treatment of polyps in CRC patients (117). It was observed in a large-scale clinical trial that the continuous use of NSAIDs has a potential to reduce the risks associated with CRC (118). A low dose of aspirin can reduce the chances of polyp recurrence by $40-50 \%$ in CRC survivors $(119,120)$. Also, NSAIDs work as antitumor drugs by inhibiting Wnt/B-catenin pathway (121). In accordance with a prior study, prostaglandin E receptor 2 (PTGER2) enhances the levels of several proinflammatory cytokines by activating NF-kB pathway in cultured neutrophils. So, the expression of IL-6, TNF- $\alpha, C X C L 1, C O X-2$ and Wnt5A increased in wild-type as compared to PTGER2 knockout mice (122). Hence, NSAIDs and PTGER2 antagonists may serve as potential preventive and therapeutic options against $F$. nucleatum-associated CRC.

Another option to treat $F$. nucleatum-associated CRC is by the use of antibiotics specific for this gram-negative bacillus. It was shown in CRC xenograft mouse model that metronidazole is effective in reducing both the $F$. nucleatum load as well as proliferation and growth of CRC tumors (123). These results suggest the use of particular antibiotics in CRC patients to reduce the burden of $F$. nucleatum. It is preferred to use the targeted narrow-spectrum antibiotics which do not disturb the normal gut flora for the treatment of $F$. nucleatum in CRC (124).

Immunotherapy is an emerging field to treat different types of tumors, based on various immune checkpoint inhibitors for cytotoxic T-lymphocyte-associated protein 4 (CTLA-4), programmed death-ligand 1 (PD-L1) and programmed cell death 1 (PDCD1) (125). Complex colonic flora of CRC patients reduce the efficacy of PDCD1 inhibitors (126). Pembrolizumab, a PDCD1 inhibitor, was approved by the Food and Drug Authority (FDA) for the treatment of MSI-high solid tumors. As $F$. nucleatum-associated CRC is characterized by MSI-high, CIMP and BRAF mutations, hence PDCD1 inhibitors may be worthwhile for treating $F$. nucleatum-positive $C R C$ cases $(42,127)$. Certainly, the use of PDCD1 inhibitors is limited due to a number of gastrointestinal side effects (128). Additionally, F. nucleatum uses its Fap2 virulence factor for binding with TIGIT receptor of T cells and NK cells and inhibits their antitumor function. In this respect, development of the anti-Fap2 antibody may play an efficient role in restoring the antitumor immune responses (64). Moreover, it was observed that the inhibition of miRNA-21 results in the reduction of metastasis of CRC cells (129). Recently, host-pathogen protein-protein interactions (HP-PPIs) clarified that there are 186 interactions between $F$. nucleatum and CRC-related host proteins that are contributed by 103 host proteins and 76 F. nucleatum proteins. Consequently, F. nucleatum-associated CRC can be treated by developing the drugs which can specifically target these HP-PPI (130).

\section{CONCLUSION}

F. nucleatum has been known to be involved in the pathogenesis of several human diseases including CRC. F. nucleatum is present in the abundance in CRC tissues and is linked with the poor prognosis of the disease. F. nucleatum invades into the mucosal epithelium through its virulence factors such as FadA and Fap2. Then $F$. nucleatum suppresses the host immune system through various molecular pathways and promotes progression of CRC. Chemoresistance is majorly observed in $F$. nucleatum-associated CRC, that results in reducing the efficacy of CRC chemotherapy. F. nucleatum can be used as a prognostic and therapeutic marker for CRC. Until now, there is no clinically available drug to treat $F$. nucleatum-associated CRC. Therefore, it is the need of the hour to design a targeted immunotherapy with minimal side effects for the efficient treatment of $F$. nucleatum-associated CRC.

\section{ACKNOWLEDGEMENTS}

This research was supported by the 2021 scientific promotion program funded by Jeju National University. 


\section{REFERENCES}

1) Tam SY, Wu VWC. A Review on the Special Radiotherapy Techniques of Colorectal Cancer. Front Onco/2019;9:208.

2) Torre LA, Bray F, Siegel RL, Ferlay J, Lortet-Tieulent J, Jemal A. Global cancer statistics, 2012. CA Cancer J Clin 2015:65:87-108.

3) Brenner H, Kloor M, Pox CP. Colorectal cancer. Lancet 2014;383:1490-502.

4) Holmes E, Li JV, Marchesi JR, Nicholson JK. Gut microbiota composition and activity in relation to host metabolic phenotype and disease risk. Cell Metab 2012;16:559-64.

5) Xu J, Gordon J. Honor thy symbionts. Proc Natl Acad Sci U S A 2003:100:10452-9.

6) Rea D, Coppola G, Palma G, Barbieri A, Luciano A, Del Prete P, et al. Microbiota effects on cancer: from risks to therapies. Oncotarget 2018:9:17915-27.

7) Arthur JC, Perez-Chanona E, Mühlbauer M, Tomkovich S, Uronis JM, Fan TJ, et al. Intestinal inflammation targets cancer-inducing activity of the microbiota. Science 2012;338:120-3.

8) Boleij A, van Gelder MM, Swinkels DW, Tjalsma H. Clinical Importance of Streptococcus gallolyticus infection among colorectal cancer patients: systematic review and meta-analysis. Clin Infect Dis 2011:53:870-8.

9) Flanagan L, Schmid J, Ebert M, Soucek P, Kunicka T, Liska V, et al. Fusobacterium nucleatum associates with stages of colorectal neoplasia development, colorectal cancer and disease outcome. Eur J Clin Microbio/ Infect Dis 2014:33:1381-90.

10) Gagnière J, Raisch J, Veziant J, Barnich N, Bonnet R, Buc E, et al. Gut microbiota imbalance and colorectal cancer. World J Gastroentero/ 2016:22:501-18.

11) Krishnan S, Eslick GD. Streptococcus bovis infection and colorectal neoplasia: a meta-analysis. Colorectal Dis 2014; 16:672-80.

12) Tsai CE, Chiu CT, Rayner CK, Wu KL, Chiu YC, Hu ML, et al. Associated factors in Streptococcus bovis bacteremia and colorectal cancer. Kaohsiung J Med Sci2016:32:196-200.

13) Viljoen KS, Dakshinamurthy A, Goldberg P, Blackburn JM. Quantitative profiling of colorectal cancer-associated bacteria reveals associations between fusobacterium spp., enterotoxigenic Bacteroides fragilis (ETBF) and clinicopathological features of colorectal cancer. PLoS One 2015;10:e0119462.

14) Zhou Y, He H, Xu H, Li Y, Li Z, Du Y, et al. Association of oncogenic bacteria with colorectal cancer in South China. Oncotarget 2016:7:80794-802.

15) Fukugaiti MH, Ignacio A, Fernandes MR, Ribeiro Júnior $U$, Nakano $V$, Avila-Campos MJ. High occurrence of Fusobacterium nucleatum and Clostridium difficile in the intestinal microbiota of colorectal carcinoma patients. Braz J Microbio/2015:46:1135-40.

16) Ito $M$, Kanno $S$, Nosho $K$, Sukawa $Y$, Mitsuhashi $K$, Kurihara $H$, et al. Association of Fusobacterium nucleatum with clinical and molecular features in colorectal serrated pathway. Int J Cancer 2015:137:1258-68.

17) Quah SY, Bergenholtz G, Tan KS. Fusobacterium nucleatum induces cytokine production through Toll-likereceptor-independent mechanism. Int Endod J 2014;47:550-9.

18) Yu T, Guo F, Yu Y, Sun T, Ma D, Han J, et al. Fusobacterium nucleatum Promotes Chemoresistance to Colorectal Cancer by Modulating Autophagy. Cel/2017:170:548-63.

19) Kostic AD, Chun E, Robertson L, Glickman JN, Gallini CA, Michaud M, et al. Fusobacterium nucleatum potentiates intestinal tumorigenesis and modulates the tumor-immune microenvironment. Cell Host Microbe 2013:14:207-15.

20) Sears $\mathrm{CL}$, Geis AL, Housseau F. Bacteroides fragilis subverts mucosal biology: from symbiont to colon carcinogenesis. 
J Clin Invest 2014;124:4166-72.

21) Wu S, Rhee KJ, Albesiano E, Rabizadeh $S, W u X$, Yen HR, et al. A human colonic commensal promotes colon tumorigenesis via activation of T helper type $17 \mathrm{~T}$ cell responses. Nat Med 2009;15:1016-22.

22) Goodwin AC, Destefano Shields CE, Wu S, Huso DL, Wu X, Murray-Stewart TR, et al. Polyamine catabolism contributes to enterotoxigenic Bacteroides fragilis-induced colon tumorigenesis. Proc Natl Acad Sci U S A 2011:108:15354-9.

23) Fujii R, Saito Y, Tokura Y, Nakagawa KI, Okuda K, Ishihara K. Characterization of bacterial flora in persistent apical periodontitis lesions. Oral Microbiol Immuno/ 2009;24:502-5.

24) Kistler JO, Booth V, Bradshaw DJ, Wade WG. Bacterial community development in experimental gingivitis. PLoS One 2013:8:e71227.

25) Yang NY, Zhang Q, Li JL, Yang SH, Shi Q. Progression of periodontal inflammation in adolescents is associated with increased number of Porphyromonas gingivalis, Prevotella intermedia, Tannerella forsythensis, and Fusobacterium nucleatum. Int J Paediatr Dent 2014:24:226-33.

26) Han YW, Fardini Y, Chen C, lacampo KG, Peraino VA, Shamonki JM, et al. Term stillbirth caused by oral Fusobacterium nucleatum. Obstet Gyneco/2010:115:442-5.

27) Kai A, Cooke F, Antoun N, Siddharthan C, Sule O. A rare presentation of ventriculitis and brain abscess caused by Fusobacterium nucleatum. J Med Microbio/2008;57:668-71.

28) Mitsuhashi $K$, Nosho $K$, Sukawa $Y$, Matsunaga $Y$, Ito $M$, Kurihara $H$, et al. Association of Fusobacterium species in pancreatic cancer tissues with molecular features and prognosis. Oncotarget 2015;6:7209-20.

29) Yoneda M, Kato S, Mawatari H, Kirikoshi H, Imajo K, Fujita K, et al. Liver abscess caused by periodontal bacterial infection with Fusobacterium necrophorum. Hepatol Res 2011:41:194-6.

30) Kostic AD, Gevers D, Pedamallu CS, Michaud M, Duke F, Earl AM, et al. Genomic analysis identifies association of Fusobacterium with colorectal carcinoma. Genome Res 2012;22:292-8.

31) Repass J, Maherali N, Owen K. Registered report: Fusobacterium nucleatum infection is prevalent in human colorectal carcinoma. Elife 2016:5:e10012.

32) Yang $Y$, Weng W, Peng J, Hong L, Yang L, Toiyama $Y$, et al. Fusobacterium nucleatum Increases Proliferation of Colorectal Cancer Cells and Tumor Development in Mice by Activating Toll-Like Receptor 4 Signaling to Nuclear Factor-kB, and Up-regulating Expression of MicroRNA-21. Gastroenterology 2017:152:851-66.

33) Warren RL, Freeman DJ, Pleasance S, Watson P, Moore RA, Cochrane K, et al. Co-occurrence of anaerobic bacteria in colorectal carcinomas. Microbiome 2013:1:16.

34) Yu J, Feng Q, Wong SH, Zhang $D$, Liang QY, Qin $Y$, et al. Metagenomic analysis of faecal microbiome as a tool towards targeted non-invasive biomarkers for colorectal cancer. Gut 2017:66:70-8.

35) Castellarin M, Warren RL, Freeman JD, Dreolini L, Krzywinski M, Strauss J, et al. Fusobacterium nucleatum infection is prevalent in human colorectal carcinoma. Genome Res 2012:22:299-306.

36) Marchesi JR, Dutilh BE, Hall N, Peters WH, Roelofs R, Boleij A, et al. Towards the human colorectal cancer microbiome. PLoS One 2011;6:e20447.

37) Li YY, Ge QX, Cao J, Zhou YJ, Du YL, Shen B, et al. Association of Fusobacterium nucleatum infection with colorectal cancer in Chinese patients. World J Gastroentero/2016:22:3227-33.

38) McCoy AN, Araújo-Pérez F, Azcárate-Peril A, Yeh JJ, Sandler RS, Keku TO. Fusobacterium is associated with colorectal adenomas. PLoS One 2013;8:e53653.

39) Mima K, Sukawa Y, Nishihara R, Qian ZR, Yamauchi M, Inamura K, et al. Fusobacterium nucleatum and T Cells in Colorectal Carcinoma. JAMA Onco/2015;1:653-61. 
40) Wong SH, Kwong TNY, Chow TC, Luk AKC, Dai RZW, Nakatsu G, et al. Quantitation of faecal Fusobacterium improves faecal immunochemical test in detecting advanced colorectal neoplasia. Gut 2017:66:1441-8.

41) Mima K, Nishihara R, Qian ZR, Cao Y, Sukawa Y, Nowak JA, et al. Fusobacterium nucleatum in colorectal carcinoma tissue and patient prognosis. Gut 2016:65:1973-80.

42) Tahara T, Yamamoto E, Suzuki H, Maruyama R, Chung W, Garriga J, et al. Fusobacterium in colonic flora and molecular features of colorectal carcinoma. Cancer Res 2014;74:1311-8.

43) Rubinstein MR, Wang X, Liu W, Hao Y, Cai G, Han YW. Fusobacterium nucleatum promotes colorectal carcinogenesis by modulating E-cadherin/B-catenin signaling via its FadA adhesin. Cell Host Microbe 2013;14:195-206.

44) Nosho K, Sukawa $Y$, Adachi $Y$, Ito M, Mitsuhashi K, Kurihara H, et al. Association of Fusobacterium nucleatum with immunity and molecular alterations in colorectal cancer. World J Gastroentero/2016:22:557-66.

45) Tjalsma H, Boleij A, Marchesi JR, Dutilh BE. A bacterial driver-passenger model for colorectal cancer: beyond the usual suspects. Nat Rev Microbio/2012;10:575-82.

46) Allen-Vercoe E, Strauss J, Chadee K. Fusobacterium nucleatum: an emerging gut pathogen? Gut Microbes 2011; 2:294-8.

47) Shang FM, Liu HL. Fusobacterium nucleatum and colorectal cancer: A review. World J Gastrointest Onco/2018;10:71-81.

48) Xie G, Huang J, Zhang Q, Han N, Shi C, Wang X. Development and validation of a TaqMan ${ }^{\text {TM }}$ fluorescent quantitative real-time PCR assay for the rapid detection of Edwardsiella tarda. Acta Oceanol Sin 2012;3:140-8.

49) Suehiro Y, Sakai K, Nishioka M, Hashimoto S, Takami T, Higaki S, et al. Highly sensitive stool DNA testing of Fusobacterium nucleatum as a marker for detection of colorectal tumours in a Japanese population. Ann Clin Biochem 2017:54:86-91.

50) Mima K, Cao Y, Chan AT, Qian ZR, Nowak JA, Masugi Y, et al. Fusobacterium nucleatum in Colorectal Carcinoma Tissue According to Tumor Location. Clin Trans/ Gastroentero/2016:7:e200.

51) Jiao H, Weng WC, Wang FJ, Cheng G, Wang W, Xie J. [Faster detection of Vibrio parahaemolyticus in foods by FQ-PCR technique]. Wei Sheng Yan Jiu. 2005:34:457-60.

52) Singh G, Sithebe A, Enitan AM, Kumari S, Bux F, Stenström TA. Comparison of droplet digital PCR and quantitative PCR for the detection of Salmonella and its application for river sediments. J Water Health 2017;15:505-8.

53) Koi M, Okita Y, Carethers JM. Fusobacterium nucleatum Infection in Colorectal Cancer: Linking Inflammation, DNA Mismatch Repair and Genetic and Epigenetic Alterations. J Anus Rectum Colon 2018:2:37-46.

54) Zhang S, Cai S, Ma Y. Association between Fusobacterium nucleatum and colorectal cancer: Progress and future directions. J Cancer 2018:9:1652-9.

55) Coppenhagen-Glazer S, Sol A, Abed J, Naor R, Zhang X, Han YW, et al. Fap2 of Fusobacterium nucleatum is a galactose-inhibitable adhesin involved in coaggregation, cell adhesion, and preterm birth. Infect Immun 2015; 83:1104-13.

56) Han YW. Fusobacterium nucleatum: a commensal-turned pathogen. Curr Opin Microbio/2015:23:141-7.

57) Liu H, Redline RW, Han YW. Fusobacterium nucleatum induces fetal death in mice via stimulation of TLR4-mediated placental inflammatory response. J Immuno/2007;179:2501-8.

58) Fardini $Y$, Wang $X$, Témoin S, Nithianantham S, Lee D, Shoham M, et al. Fusobacterium nucleatum adhesin FadA binds vascular endothelial cadherin and alters endothelial integrity. Mol Microbio/2011:82:1468-80.

59) Témoin S, Wu KL, Wu V, Shoham M, Han YW. Signal peptide of FadA adhesin from Fusobacterium nucleatum plays a novel structural role by modulating the filament's length and width. FEBS Lett 2012:586:1-6. 
60) Xu M, Yamada M, Li M, Liu H, Chen SG, Han YW. FadA from Fusobacterium nucleatum utilizes both secreted and nonsecreted forms for functional oligomerization for attachment and invasion of host cells. J Biol Chem 2007;282:25000-9.

61) Chen $Y$, Peng $Y, Y u$ J, Chen $T$, Wu Y, Shi L, et al. Invasive Fusobacterium nucleatum activates beta-catenin signaling in colorectal cancer via a TLR4/P-PAK1 cascade. Oncotarget 2017:8:31802-14.

62) Abed J, Emgård JE, Zamir G, Faroja M, Almogy G, Grenov A, et al. Fap2 Mediates Fusobacterium nucleatum Colorectal Adenocarcinoma Enrichment by Binding to Tumor-Expressed Gal-GalNAc. Cell Host Microbe 2016;20:215-25.

63) Chauvin JM, Zarour HM. TIGIT in cancer immunotherapy. J Immunother Cancer 2020;8:e000957.

64) Gur C, Ibrahim Y, Isaacson B, Yamin R, Abed J, Gamliel M, et al. Binding of the Fap2 protein of Fusobacterium nucleatum to human inhibitory receptor TIGIT protects tumors from immune cell attack. Immunity 2015;42:344-55

65) Phipps Al, Limburg PJ, Baron JA, Burnett-Hartman AN, Weisenberger DJ, Laird PW, et al. Association between molecular subtypes of colorectal cancer and patient survival. Gastroenterology 2015:148:77-87.

66) Yamauchi M, Morikawa T, Kuchiba A, Imamura Y, Qian ZR, Nishihara R, et al. Assessment of colorectal cancer molecular features along bowel subsites challenges the conception of distinct dichotomy of proximal versus distal colorectum. Gut 2012;61:847-54.

67) Leung A, Tsoi H, Yu J. Fusobacterium and Escherichia: models of colorectal cancer driven by microbiota and the utility of microbiota in colorectal cancer screening. Expert Rev Gastroenterol Hepatol 2015:9:651-7.

68) Toiyama Y, Okugawa Y, Goel A. DNA methylation and microRNA biomarkers for noninvasive detection of gastric and colorectal cancer. Biochem Biophys Res Commun 2014;455:43-57.

69) Merline R, Moreth K, Beckmann J, Nastase MV, Zeng-Brouwers J, Tralhão JG, et al. Signaling by the matrix proteoglycan decorin controls inflammation and cancer through PDCD4 and MicroRNA-21. Sci Signa/2011;4:ra75.

70) Sheedy FJ, Palsson-McDermott E, Hennessy EJ, Martin C, O'Leary JJ, Ruan Q, et al. Negative regulation of TLR4 via targeting of the proinflammatory tumor suppressor PDCD4 by the microRNA miR-21. Nat Immuno/2010;11:141-7.

71) Schetter AJ, Leung SY, Sohn JJ, Zanetti KA, Bowman ED, Yanaihara N, et al. MicroRNA expression profiles associated with prognosis and therapeutic outcome in colon adenocarcinoma. JAMA 2008;299:425-36.

72) Mantovani A, Cassatella MA, Costantini C, Jaillon S. Neutrophils in the activation and regulation of innate and adaptive immunity. Nat Rev Immuno/2011:11:519-31.

73) Edin S, Wikberg ML, Dahlin AM, Rutegård J, Öberg A, Oldenborg PA, et al. The distribution of macrophages with a $\mathrm{M} 1$ or $\mathrm{M} 2$ phenotype in relation to prognosis and the molecular characteristics of colorectal cancer. PLoS One 2012:7:e47045.

74) Chen T, Li Q, Wu J, Wu Y, Peng W, Li H, et al. Fusobacterium nucleatum promotes M2 polarization of macrophages in the microenvironment of colorectal tumours via a TLR4-dependent mechanism. Cancer Immunol Immunother 2018;67:1635-46.

75) Abe K. Butyric acid induces apoptosis in both human monocytes and lymphocytes equivalently. J Oral Sci 2012:54:7-14.

76) Xue Y, Xiao H, Guo S, Xu B, Liao Y, Wu Y, et al. Indoleamine 2,3-dioxygenase expression regulates the survival and proliferation of Fusobacterium nucleatum in THP-1-derived macrophages. Cell Death Dis 2018:9:355.

77) Kaplan CW, Ma X, Paranjpe A, Jewett A, Lux R, Kinder-Haake S, et al. Fusobacterium nucleatum outer membrane proteins Fap2 and RadD induce cell death in human lymphocytes. Infect Immun 2010;78:4773-8.

78) Chen T, Li Q, Zhang X, Long R, Wu Y, Wu J, et al. TOX expression decreases with progression of colorectal cancers and is associated with CD4 T-cell density and Fusobacterium nucleatum infection. Hum Patho/2018;79:93-101.

79) Huynh T, Kapur RV, Kaplan CW, Cacalano N, Kinder Haake S, Shi W, et al. The role of aggregation in Fusobacterium 
nucleatum- induced immune cell death. J Endod 2011;37:1531-5.

80) Shenker BJ, Datar S. Fusobacterium nucleatum inhibits human T-cell activation by arresting cells in the mid-G1 phase of the cell cycle. Infect Immun 1995:63:4830-6.

81) Shenker BJ, DiRienzo JM. Suppression of human peripheral blood lymphocytes by Fusobacterium nucleatum. $J$ Immunol 1984;132:2357-62.

82) Bashir A, Miskeen AY, Hazari YM, Asrafuzzaman S, Fazili KM. Fusobacterium nucleatum, inflammation, and immunity: the fire within human gut. Tumour Bio/2016:37:2805-10.

83) Guevarra LA Jr, Afable ACF, Belza PJO, Dy KJS, Lee SJQ, Sy-Ortin TT, et al. Immunogenicity of a Fap2 peptide mimotope of Fusobacterium nucleatum and its potential use in the diagnosis of colorectal cancer. Infect Agent Cancer2018;13:11.

84) Coombes JL, Siddiqui KR, Arancibia-Cárcamo CV, Hall J, Sun CM, Belkaid Y, et al. A functionally specialized population of mucosal CD103+ DCs induces Foxp3+ regulatory T cells via a TGF-beta and retinoic acid-dependent mechanism. J Exp Med 2007;204:1757-64.

85) Rao HL, Chen JW, Li M, Xiao YB, Fu J, Zeng YX, et al. Increased intratumoral neutrophil in colorectal carcinomas correlates closely with malignant phenotype and predicts patients' adverse prognosis. PLoS One 2012:7:e30806.

86) Seow HF, Yip WK, Fifis T. Advances in targeted and immunobased therapies for colorectal cancer in the genomic era. Onco Targets Ther 2016:9:1899-920.

87) Temraz S, Mukherji D, Alameddine R, Shamseddine A. Methods of overcoming treatment resistance in colorectal cancer. Crit Rev Oncol Hemato/2014;89:217-30.

88) Sun Y. Tumor microenvironment and cancer therapy resistance. Cancer Lett 2016:380:205-15.

89) Ulivi P, Marisi G, Passardi A. Relationship between hypoxia and response to antiangiogenic therapy in metastatic colorectal cancer. Oncotarget 2016:7:46678-91.

90) Feng PH, Yu CT, Chen KY, Luo CS, Wu SM, Liu CY, et al. S100A9(+) MDSC and TAM-mediated EGFR-TKI resistance in lung adenocarcinoma: the role of RELB. Oncotarget 2018;9:7631-43.

91) Romano A, Parrinello NL, La Cava P, Tibullo D, Giallongo C, Camiolo G, et al. PMN-MDSC and arginase are increased in myeloma and may contribute to resistance to therapy. Expert Rev Mol Diagn 2018;18:675-83.

92) Rex DK, Johnson DA, Anderson JC, Schoenfeld PS, Burke CA, Inadomi JM. American College of Gastroenterology guidelines for colorectal cancer screening 2009 [corrected]. Am J Gastroentero/ 2009;104:739-50.

93) Sung JJ, Ng SC, Chan FK, Chiu HM, Kim HS, Matsuda T, et al. An updated Asia Pacific Consensus Recommendations on colorectal cancer screening. Gut 2015:64:121-32.

94) Amitay EL, Werner S, Vital M, Pieper DH, Höfler D, Gierse IJ, et al. Fusobacterium and colorectal cancer: causal factor or passenger? Results from a large colorectal cancer screening study. Carcinogenesis 2017:38:781-8.

95) Kurt M, Yumuk Z. Diagnostic accuracy of Fusobacterium nucleatum IgA and IgG ELISA test in colorectal cancer. Sci Rep. 2021:11:1608.

96) Wang HF, Li LF, Guo SH, Zeng QY, Ning F, Liu WL, et al. Evaluation of antibody level against Fusobacterium nucleatum in the serological diagnosis of colorectal cancer. Sci Rep 2016:6:33440.

97) Hundt S, Haug U, Brenner H. Comparative evaluation of immunochemical fecal occult blood tests for colorectal adenoma detection. Ann Intern Med 2009;150:162-9.

98) Tunsjø HS, Gundersen G, Rangnes F, Noone JC, Endres A, Bemanian V. Detection of Fusobacterium nucleatum in stool and colonic tissues from Norwegian colorectal cancer patients. Eur J Clin Microbiol Infect Dis 2019;38:1367-76. 
99) Huang S, Yang Z, Zou D, Dong D, Liu A, Liu W, et al. Rapid detection of nusG and fadA in Fusobacterium nucleatum by loop-mediated isothermal amplification. J Med Microbio/2016:65:760-9.

100) Liu L, Tabung FK, Zhang X, Nowak JA, Qian ZR, Hamada T, et al. Diets That Promote Colon Inflammation Associate With Risk of Colorectal Carcinomas That Contain Fusobacterium nucleatum. Clin Gastroenterol Hepato/2018;16:1622-31.

101) Tabung FK, Liu L, Wang W, Fung TT, Wu K, Smith-Warner SA, et al. Association of Dietary Inflammatory Potential With Colorectal Cancer Risk in Men and Women. JAMA Onco/2018;4:366-73.

102) Berg A. Nutrition, development, and population growth. Popul Bull 1973;29:3-37.

103) Mafiana RN, Al Lawati AS, Waly MI, Al Farsi Y, Al Kindi M, Al Moundhri M. Association between Dietary and Lifestyle Indices and Colorectal Cancer in Oman: A Case-Control Study. Asian Pac J Cancer Prev 2018;19:3117-22.

104) Song M, Garrett WS, Chan AT. Nutrients, foods, and colorectal cancer prevention. Gastroenterology 2015;148:1244-60.

105) Ali I, Koh YS. High-fat-diet-modulated Gut Microbiota Promotes Intestinal Carcinogenesis. J Bacterio/ Virol 2015:45:394-6.

106) David LA, Maurice CF, Carmody RN, Gootenberg DB, Button JE, Wolfe BE, et al. Diet rapidly and reproducibly alters the human gut microbiome. Nature 2014;505:559-63.

107) Xu Z, Knight R. Dietary effects on human gut microbiome diversity. Br J Nutr 2015:113:S1-5.

108) Tabung FK, Smith-Warner SA, Chavarro JE, Wu K, Fuchs CS, Hu FB, et al. Development and Validation of an Empirical Dietary Inflammatory Index. J Nutr 2016:146:1560-70.

109) O’Keefe SJ, Li JV, Lahti L, Ou J, Carbonero F, Mohammed K, et al. Fat, fibre and cancer risk in African Americans and rural Africans. Nat Commun. 2015;6:6342.

110) Mehta RS, Nishihara R, Cao Y, Song M, Mima K, Qian ZR, et al. Association of dietary patterns with risk of colorectal cancer subtypes classified by Fusobacterium nucleatum in tumor tissue. JAMA Onco/2017:3:921-7.

111) Dharmani P, Strauss J, Ambrose C, Allen-Vercoe E, Chadee K. Fusobacterium nucleatum infection of colonic cells stimulates MUC2 mucin and tumor necrosis factor alpha. Infect Immun 2011;79:2597-607.

112) Taniguchi K, Karin M. NF-kB, inflammation, immunity and cancer: coming of age. Nat Rev Immuno/2018;18:309-24

113) Trinchieri G. Cancer and inflammation: an old intuition with rapidly evolving new concepts. Annu Rev Immunol 2012;30:677-706.

114) Lasry A, Zinger A, Ben-Neriah Y. Inflammatory networks underlying colorectal cancer. Nat Immuno/2016:17:230-40.

115) Williams CS, Shattuck-Brandt RL, DuBois RN. The role of COX-2 in intestinal cancer. Expert Opin Investig Drugs 1999:8:1-12.

116) Huang WW, Hsieh KP, Huang RY, Yang YH. Role of cyclooxygenase-2 inhibitors in the survival outcome of colorectal cancer patients: A population-based cohort study. Kaohsiung J Med Sci 2017:33:308-14.

117) Waddell WR, Ganser GF, Cerise EJ, Loughry RW. Sulindac for polyposis of the colon. Am J Surg 1989;157:175-9.

118) Rothwell PM, Wilson M, Elwin CE, Norrving B, Algra A, Warlow CP, et al. Long-term effect of aspirin on colorectal cancer incidence and mortality: 20-year follow-up of five randomised trials. Lancet 2010;376:1741-50.

119) Johnson CC, Jankowski M, Rolnick S, Yood MU, Alford SH. Influence of NSAID Use Among Colorectal Cancer Survivors on Cancer Outcomes. Am J Clin Oncol 2017:40:370-4.

120) Tsoi KK, Chan FC, Hirai HW, Sung JJ. Risk of gastrointestinal bleeding and benefit from colorectal cancer reduction from long-term use of low-dose aspirin: A retrospective study of 612509 patients. J Gastroentero/ Hepato/2018;33:1728-36.

121) Bos CL, Kodach LL, van den Brink GR, Diks SH, van Santen MM, Richel DJ, et al. Effect of aspirin on the Wnt/beta-catenin pathway is mediated via protein phosphatase 2A. Oncogene 2006;25:6447-56. 
122) Ma X, Aoki T, Tsuruyama T, Narumiya S. Definition of Prostaglandin E2-EP2 Signals in the Colon Tumor Microenvironment That Amplify Inflammation and Tumor Growth. Cancer Res 2015:75:2822-32.

123) Bullman S, Pedamallu CS, Sicinska E, Clancy TE, Zhang X, Cai D, et al. Analysis of Fusobacterium persistence and antibiotic response in colorectal cancer. Science 2017;358:1443-8.

124) Haak BW, Lankelma JM, Hugenholtz F, Belzer C, de Vos WM, Wiersinga WJ. Long-term impact of oral vancomycin, ciprofloxacin and metronidazole on the gut microbiota in healthy humans. J Antimicrob Chemother 2019;74:782-6.

125) Bever KM, Le DT. An Expanding Role for Immunotherapy in Colorectal Cancer. J Nat/ Compr Canc Netw 2017:15:401-10.

126) Bhatt AP, Redinbo MR, Bultman SJ. The role of the microbiome in cancer development and therapy. CA Cancer J Clin 2017:67:326-44.

127) Chakravarty D, Gao J, Phillips SM, Kundra R, Zhang H, Wang J, et al. OncoKB: a precision oncology knowledge base. JCO Precis Oncol2017;2017: PO.17.00029.

128) Cramer P, Bresalier RS. Gastrointestinal and Hepatic Complications of Immune Checkpoint Inhibitors. Curr Gastroenterol Rep 2017;19:3.

129) Nedaeinia R, Sharifi M, Avan A, Kazemi M, Nabinejad A, Ferns GA, et al. Inhibition of microRNA-21 via locked nucleic acid-anti-miR suppressed metastatic features of colorectal cancer cells through modulation of programmed cell death 4. Tumour Bio/2017;39:1010428317692261.

130) Kumar A, Thotakura PL, Tiwary BK, Krishna R. Target identification in Fusobacterium nucleatum by subtractive genomics approach and enrichment analysis of host-pathogen protein-protein interactions. BMC Microbiol $2016 ; 16: 84$. 